UDC 512.5

B. A. F. Wehrfritz (School Math. Sci. Queen Mary, Univ. London, England)

\title{
FINITARY GROUPS AND KRULL DIMENSION OVER THE INTEGERS
}

\section{ФІНІТАРНІ ГРУПИ І РОЗМІРНІСТЬ КРУЛЛЯ НАД ЦІЛИМИ ЧИСЛАМИ}

Let $M$ be any abelian group. We make a detailed study for reasons explained in the Introduction of the normal subgroup

$$
F_{\infty} \text { Aut } M=\{g \in \text { Aut } M: M(g-1) \text { is a minimax group }\}
$$

of the automorphism group Aut $M$ of $M$. The conclusions, although slightly weaker than one would hope, in that they do not fully explain the common behaviour of the finitary and the Artinian-finitary subgroups of Aut $M$, are certainly stronger that one might reasonably expect. Our main focus is on residual properties and unipotence.

Нехай $M$ - будь-яка абелева група. 3 мотивів, що вказані у вступі до статті, детально вивчено нормальну підгрупу

$$
F_{\infty} \text { Aut } M=\{g \in \text { Aut } M: M(g-1) \text { - мінімаксна група }\}
$$

групи Aut $M$ автоморфізмів $M$. Хоча отримані результати слабкіші, ніж хотілося б, оскільки вони не повністю пояснюють загальну поведінку фінітарних та артіново-фінітарних підгруп групи Aut $M$, але вони беззаперечно сильніші, ніж можна було б очікувати. Головну увагу приділено залишковим властивостям та уніпотентності.

1. Introduction. For $M$ an abelian group we study in this paper the normal subgroup

$$
F_{\infty} \text { Aut } M=\{g \in \text { Aut } M: M(g-1) \text { has Krull dimension over the integers }\}
$$

of the automorphism group Aut $M$ of $M$. On Page 255 of Vol. 2 of this book [1] Fuchs appeals for a greater understanding of the automorphism group of an abelian group and in particular of the normal subgroups of such an automorphism group. This paper could be interpreted as a small contribution to this appeal.

However, our motivation has come from more general ring and module theoretic considerations. If $M$ is any module over the commutative ring $R$, set

$$
\begin{aligned}
& F \text { Aut }_{R} M=\left\{g \in \operatorname{Aut}_{R} M: M(g-1) \text { is } R \text {-Noetherian }\right\} \\
& F_{1} \text { Aut }_{R} M=\left\{g \in \text { Aut }_{R} M: M(g-1) \text { is } R \text {-Artinian }\right\}
\end{aligned}
$$

and

$$
F_{\infty} \operatorname{Aut}_{R} M=\left\{g \in \operatorname{Aut}_{R} M: M(g-1) \text { has Krull dimension as } R \text {-module }\right\} .
$$

These are three examples of an infinite number of (generalized) finitary groups, the first being the finitary group and the second the Artinian-finitary group. They are almost the only finitary groups relevant to this paper, but for accounts of the general set-up, see [2] and [3], Section 2.

Now $F$ Aut $_{R} M$ and $F_{1}$ Aut $_{R} M$ are both subgroups of $F_{\infty}$ Aut ${ }_{R} M$. Also they have very many properties in common, see [4 - 8] and especially [9]. Now do these properties come from a common factor? Specifically, do they have these properties in common because $F_{\infty}$ Aut $_{R} M$ also enjoys these properties? We propose to study this question here just in the special case where $R$ is the ring $Z$ of the integers. The answer it turns out in this case is frequently no, but then $F_{\infty}$ Aut $M$ only just avoids satisfying them. While working over the integers we suppress the suffix $\boldsymbol{Z}$ and just 
write $F$ Aut $M, F_{1}$ Aut $M$ and $F_{\infty}$ Aut $M$. The first two of these three groups we have already investigated in some detail in [5]. Thus this paper is devoted the third.

Notationally it is convenient in this Introduction to include the possibility of an arbitrary commutative ring $R$. Thus just throughout this section $R$ denotes a commutative ring and $M$ an $R$-module. Mostly we only consider the additive structure, that is the $Z$-module structure, of $M$ and hence we mainly refer to $F$ Aut $M$ rather than $F$ Aut $_{R} M$, and similarly with the other finitary groups.

Suppose $M$ has Krull dimension over $\boldsymbol{Z}$. Then every section of $M$ has finite uniform dimension and so $M$ has a free abelian subgroup of finite rank modulo which $M$ is a direct sum of a finite number of finite cyclic groups and Prüfer groups. Such a group is usually called a minimax group. Conversely, a minimax group is Noetherian by Artinian and has Krull dimension (at most one). Thus we propose to call $F_{\infty}$ Aut $M$ the minimax-finitary group of $M$ (over $\boldsymbol{Z}$ ).

We will see below, and it is very easy, that there is a natural exact sequence

$$
1 \rightarrow F_{1} \text { Aut } M \rightarrow F_{\infty} \text { Aut } M \rightarrow F G L(Q \otimes M) .
$$

Of course $\boldsymbol{Q}$ denotes the rationals. If $V$ is a vector space over some field $F$, then $F G L(V)$ denotes the finitary general linear group over $V$. Thus $F_{\infty}$ Aut $M$ is Artinian-finitary by finitary-linear. The proof also yields that if $T$ denotes the $Z$ torsion submodule of $M$, as it will throughout this paper, then $A=C_{G}(M / T) \cap$ $\cap C_{G}(T)$ is an abelian normal subgroup of $G$ such that $G / A$ embeds into $F G L(Q \otimes M) \times F_{1}$ Aut $T$. These simple remarks produce a crude reduction of the study of $F_{\infty}$ Aut $M$ to that of finitary-linear and Artinian-finitary groups. For example, it is immediate that periodic subgroups of $F_{\infty}$ Aut $M$ are locally finite. But it is a quite crude reduction; for example, it only produces a number of metabelian subgroups of $F_{\infty}$ Aut $M$, while a more careful study shows that there exist nilpotent-of-class-at-most2 (occasionally even abelian) subgroups that fulfill the same roles.

We now summarise our main results. We start with a consideration of residual properties. Both $F$ Aut $M$ and $F_{1}$ Aut $M$ are locally residually finite and also are abelian by residually locally finite $\left([5], \mathbf{1 0}^{\mathbf{1}}, \mathbf{1 0}_{\mathbf{1}}, \mathbf{1 1}^{\mathbf{1}}\right.$ and $\left.\mathbf{1 1}_{1}\right)$. Further both $F$ Aut $M$ and $F_{1}$ Aut $M$ are, modulo a central unipotent subgroup, residually (finitary over commutative Noetherian rings), see [9], Proposition 8.11. None of these hold for $F_{\infty}$ Aut $M$ as we show with examples. However, they nearly hold.

Theorem 1. Let $M$ be an abelian group.

a) $F_{\infty}$ Aut $M$ is abelian by locally residually finite and hence locally (abelian by residually finite). If $G$ is any finitely generated subgroup of $F_{\infty}$ Aut $M$, then $G$ has normal subgroups $A \leq H$ such that $A$ is abelian, $H / A$ is residually a finite nilpotent $\pi$-group for some finite set $\pi$ of primes and $G / H$ is finite.

b) $F_{\infty}$ Aut $M$ is nilpotent-of-class-at-most-2 by residually locally finite.

c) $F_{\infty}$ Aut $M$ is unipotent-abelian by residually (finitary over the rationals $\boldsymbol{Q}$ and finite images of $\boldsymbol{Z}$ ).

As we have stated the theorem, Part a) it easy. However with a little more work we can be much more precise about the set $\pi$ of primes, see the proof of 3.2. We show with examples that the group $G$ of Part a) of Theorem 1 need not be central by residually finite (let alone residually finite). Also in Part b) the periodic residual of $G \leq F_{\infty}$ Aut $M$, although nilpotent of class at most 2, need not be abelian, or central (in $G$ ) by abelian or abelian by central (in $G$ ). In Part c) the unipotent abelian normal subgroup cannot be replaced by a central subgroup of $G$. 
Any subgroup $G$ of $F_{\infty}$ Aut $M$ has a unipotent radical, which we denote by $u(G)$. Precisely what we mean by this is spelled out by the next theorem. For the latter parts of this theorem we need some further notation. By $\psi$ we denote the natural map of $F_{\infty}$ Aut $M$ into $F G L(Q \otimes M)$ discussed above. By an $s$-subgroup of Aut $M$ (or of Aut $\left._{R} M\right)$ we mean a subgroup each of whose finitely generated subgroups stabilizes an ascending series of submodules of $M$. Unlike the unipotent radical, the $s$-radical $s(G)$ of $G$ exists under very general conditions and often has to be used as a replacement for a unipotent radical, see [2], especially Section 4. Finally 5.2 of [8] introduces a collection of maps $\psi_{\lambda}$ attached to an $R$-module $M$ and a subgroup $G$ of $F_{1}$ Aut $_{R} M$. Since we shall only user these maps once and that only in order to apply [8], 5.2, we will not burden the reader with their precise construction. See [8] for details.

Theorem 2. Let $G$ be a subgroup of $F_{\infty}$ Aut $M$. Then $G$ has a unipotent radical. Specifically $G$ has a normal subgroup $u(G)$ with the following properties.

a) $u(G)$ is a unipotent normal subgroup of $G$ containing every unipotent normal subgroup of $G$.

b) $G / u(G)$ is a subdirect product of irreducible finitary linear groups over $Q$, $G F(p)$ and $Q_{p}$, the $p$-adic numbers, for various primes $p$.

c) Let $H$ be a finitely $G$-generated normal subgroup of $G$. Then $u(H)=$ $=H \cap u(G)$ and $u(H)$ is nilpotent. In particular $u(G)$ lies in the Fitting subgroup of $G$ and $u(G)$ is a Fitting group.

d) If $H$ is an ascendent subgroup of $G$, then $u(H)$ is an ascendent subgroup of $u(G)$.

e) $u(G) \leq s(G)$ and $s(G) \psi \leq u(G \psi)$.

f) $u(G)=G \cap(u(G \psi)) \psi^{-1} \cap\left(1+\bigcap_{\lambda} \operatorname{ker} \psi_{\lambda}\right)$, where the $\psi_{\lambda}$ are the maps of [8], 5.2, with respect to the $\mathbb{Z}$-torsion submodule $T$ of $M$ and the subgroup $\left.G\right|_{T}$ of Aut $T$. (The map $\psi$ is described above.)

We can now state a further residual result. It effectively copies the corresponding results for $F$ Aut $_{R} M$ and $F_{1}$ Aut $_{R} M$, which we have previously only written out for local rings (see [9], Theorem 5), since for arbitrary commutative rings $R$ it becomes very cumbersome. The pattern, however, is clearly the same. Note that $B$ remains abelian; it does not get replaced by a subgroup that is nilpotent of class at most 2 or even one that is metabelian, as Theorem 1 might suggest. The analogues of Parts c) and g) of [9] (Theorem 5) we have incorporated into Theorem 2 above and hence have not repeated below. Part f) of Theorem 3 below has no analogue in [9], Theorem 5, although it does in [9], Theorem 6a).

Theorem 3. Let $G$ be a subgroup of $F_{\infty}$ Aut $M$. Then $G$ has a normal series $\langle 1\rangle \leq B \leq K \leq u(G) \leq G$, where

a) $B$ is abelian;

b) $K / B$ is residually (nilpotent of finite exponent);

c) $u(G) / B$ is a subdirect product of a torsion-free group and a residually periodic group; it is also abelian by residually periodic;

d) $u(G) / K$ is a subdirect product of unipotent finitary linear groups over $\boldsymbol{Q}$, $G F(p)$ and $Q_{p}$ for various primes $p$

e) $G / K$ is a subdirect product of finitary linear groups over $Q, G F(p)$ and $Q_{p}$ for various primes $p$

f) $K$ stabilizes an ascending series in $M$. 
Information about the sections $G / u(G)$ and $u(G)$ of $G$ omitted from Theorem 3 is given in Theorem 2. Our final theorem concerns the structure of unipotent groups and unipotent radicals. To state it reasonably succinctly we use the following notation for group classes and operators. $A$ denotes the class of abelian groups, $N_{2}$ the class of nilpotent groups of class at most 2, $N$ the class of nilpotent groups, $\boldsymbol{F}$ the class of finite groups and $\boldsymbol{E}$ the class of groups of finite exponent. Also if $\boldsymbol{X}$ and $\boldsymbol{Y}$ are any group classes, then $S(\boldsymbol{X} \times \boldsymbol{Y})$ denotes the class of all subgroups of direct products of an $\boldsymbol{X}$-group and a $\mathbf{Y}$-group and $L \boldsymbol{X}$ (resp. $L_{n} \boldsymbol{X}$ ) denotes the class of all groups $G$ each of whose finite subsets lies in an $\boldsymbol{X}$-subgroup (resp. normal $\boldsymbol{X}$-subgroup) of $G$.

Theorem 4. Let $G$ be a subgroup of $F_{\infty}$ Aut $M$.

a) If $G$ is unipotent, then $G$ is periodic-abelian by torsion-free by periodic.

b) Let $X$ be a finite subset of $G$ and $U$ the maximal periodic subgroup of the unipotent radical of the normal subgroup $\left\langle X^{G}\right\rangle$ of $G$ generated by $X$. Then

$$
U \in N \cap N_{2} E \cap A . S(A \times E) \leq A E A .
$$

c) If $G$ is periodic and unipotent, then

$$
G \in L_{n}\left(N \cap N_{2} E \cap A E A\right) \text { and } G^{\prime} \in L_{n}(N \cap A E) .
$$

Since in Part a) the group $G$ is locally nilpotent and locally nilpotent groups are always periodic by torsion-free, Part a) is saying that $G$ is not far from being a subgroup group of a direct product of a periodic group and a torsion-free group. Since we already know by [9], Theorem 2, that in Part a) above the conclusions hold whenever $G$ is contained in either $F$ Aut $M$ or $F_{1}$ Aut $M$, this is an instance where an abelian normal subgroup need not be replaced in the $F_{\infty}$ Aut $M$ case by, say, a nilpotent normal subgroup of class at most 2 .

In Part b) if $G$ lies in either $F$ Aut $M$ or $F_{1}$ Aut $M$ then

$$
U \in N \cap \boldsymbol{A} \boldsymbol{E} \cap \boldsymbol{E} \boldsymbol{A} \cap(\boldsymbol{A} \cap \boldsymbol{E}) . S(\boldsymbol{A} \times \boldsymbol{E})
$$

by [9], Theorem 4, so again we get a weakening in Part b) of our Theorem 4, but perhaps its from is not so predictable. Similar remarks apply to a comparison between Part c) of Theorem 4 with the Corollary to Theorem 4 of [9].

Theorem 2 is proved in Section 2 below, Theorems 1 and 3 in Section 3 and Theorem 4 in Section 4. In our final section, Section 5, we consider a variant of the above, namely that arising from considering extensions of Artinian groups by Noetherian groups rather than the reverse. This section also contains some further counter examples.

\section{General results.}

2.1. Lemma. Let $G=F_{\infty}$ Aut $M$.

a) There is a natural exact sequence $1 \rightarrow F_{1}$ Aut $M \rightarrow F_{\infty}$ Aut $M \rightarrow F G L(Q \otimes$ $\otimes M)$.

b) If $A=C_{G}(M / T) \cap C_{G}(T)$, then $A$ is abelian and $G / A$ embeds into the direct product $F G L(Q \otimes M) \times F_{1}$ Aut $T$. ( $T$ recall is the $Z$-torsion submodule of M.) In particular $G / A$ is locally quasilinear.

c) $F_{1}$ Aut $M$ embeds into $F$ Aut $_{\boldsymbol{J}} N$, where $\boldsymbol{J}=\prod_{p} \boldsymbol{Z}_{p}$ is the profinite closure of $\boldsymbol{Z}$ and $N$ is some $\boldsymbol{J}$-module.

d) Periodic subgroups of $F_{\infty}$ Aut $M$ are locally finite.

So by a) and c) the group $F_{\infty}$ Aut $M$ is an extension of a finitary group over $\boldsymbol{J}$ by a finitary group over $Q$.

Proof. a) Let $L=F_{\infty}$ Aut $M$ and $g \in G$. If $g \in F_{1}$ Aut $M$, then $M(g-1)$ is torsion and $g \in C_{G}(M / T)$. Conversely, if $g \in C_{G}(M / T)$, then $M(g-1)$ is 
minimax, torsion and hence Artinian. Thus $g \in F_{1}$ Aut $M$ and so $F_{1}$ Aut $M=$ $=C_{G}(M / T)$. Clearly $G / C_{G}(M / T)$ embeds naturally into $F_{\infty} \operatorname{Aut}(M / T)$ and $M / T$ embeds in the obvious way into $Q \otimes(M / T) \cong Q \otimes M$. This leads to an embedding of $F_{\infty} \operatorname{Aut}(M / T)$ into $F_{\infty} \operatorname{Aut}_{\boldsymbol{Q}}(\boldsymbol{Q} \otimes M)=F G L(\boldsymbol{Q} \otimes M)$. Part a) follows.

b) $G / C_{G}(M / T)$ embeds into $F G L(Q \otimes M)$ as in Part a) and $G / C_{G}(T)$ embeds into $F_{\infty}$ Aut $T=F_{1}$ Aut $T$, again see Part a). By stability theory $A$ is abelian. By N4 and A3 of [7] the group $G / A$ is locally quasilinear. Part b) follows.

c) A application of the proof of $\mathbf{A 1}$ of [7] to our $M$ yields Part c).

d) This follows from a), the corresponding results for finitary and Artinian-finitary groups ([7], N4 and A3) and the simple and well-known fact that extensions of locally finite groups by locally finite groups are locally finite.

2.2. Proof of Theorem 2. a) \& f). Define $u(G)$ by the formula in $f$ ). Certainly $u(G)$ is normal in $G$ and if $U$ is a unipotent normal subgroup of $G$, then $U \psi \leq$ $\leq u(G \psi)$ and each $U \psi_{\lambda}=\langle 1\rangle$. Thus $U \leq u(G)$.

Let $g \in u(G)$. Then $g \psi$ is unipotent, so $(g \psi-1)^{m}=0$ for some $m$. It follows from the definition of $\psi$ that $M(g-1)^{m} \leq T$. Also $g$ acts unipotently on $T$ by $\mathbf{5 . 2}$ of [8], say $T(g-1)^{n}=\{0\}$. Trivially then $(g-1)^{m+n}=0$ and so $u(G)$ is unipotent.

b) It follows from [8], 5.2, and the finitary linear case that $G / u(G)$ is a subdirect product of certain irreducible finitary linear images of $G$. We have only to specify the ground fields. From $G \psi$ only the rationals $\boldsymbol{Q}$ arise. One has to check through the proofs in [8] to see that the only ground fields that arise from the $\psi_{\lambda}$ are the $G F(p)$ and $\boldsymbol{Q}_{p}$ for various primes $p$.

c) The group $u(H)$ is normal in $G$, so $u(H) \leq H \cap u(G)$ and $H \cap u(G)$ is unipotent and normal in $H$. Therefore $u(H)=H \cap u(G)$. If $\boldsymbol{u}$ denotes the augmentation ideal of $u(H)$, then $T \boldsymbol{u}^{s}=\{0\}$ for some $s$ by [8], 5.2, and $M \boldsymbol{u}^{t} \leq T$ for some $t$ by [10], 3.7 (or the finitary linear version if you prefer). Thus $\mathbf{M u}^{s+t}=$ $=\{0\}$ and all of Part $\mathrm{c}$ ) is a consequence of this.

d) The usual transfinite induction yields this (cf. [4], 3.8, or [2], 4.10).

e) By the proof of c), if $U$ is a finitely generated subgroup of $u(G)$, then $U$ stabilizes a finite series in $M$. Hence $u(G)$ is an $s$-subgroup, so $u(G) \leq s(G)$. By 4.2 and 4.3 of [2] we have that $s(G) \psi$ is a $s$-subgroup and hence a unipotent subgroup of $F G L(Q \otimes M)$. (Note that all nontrivial torsion-free abelian groups are tight.) Thus $s(G) \psi \leq u(G \psi)$.

2.3. Remark. If $M$ is minimax, then Aut $M$ can contain a unipotent Prüfer subgroup, indeed even a noncentral one. (If $M$ is Noetherian or Artinian, then Aut $M$ contains no Prüfer subgroups.) For let $M=Z \oplus P$, where $P$ is a Prüfer $p$-group for some prime $p$. Then $P$ is isomorphic to the unipotent subgroup $Q=\left(\begin{array}{ll}1 & P \\ 0 & 1\end{array}\right)$ of Aut $M$. If $U$ is the group of units of the $p$-adic integers, then $Q$ is a noncentral Prüfer subgroup of the subgroup $G=\operatorname{diag}(1, U) . Q$ of Aut $M$. Further $G$ is not residually finite or even central by residually finite. This example is pertinent to both 2.4 and 2.5 below.

2.4. Lemma. Let $M$ be an abelian minimax group and let $\operatorname{spec} M$ denote the (finite) set of primes $p$ such that $M$ has a Prüfer p-group section. Set $G=$ = Aut $M$.

a) If $p \notin \operatorname{spec} M$, then $\bigcap_{i} p^{i} M$ is torsion. 
b) If $M$ is torsion-free then $G$ is residually finite; in fact if $p \notin \operatorname{spec} M$, then $G$ is (residually finite- $p$ ) by finite.

c) If $M$ is a p-group, then $G$ is residually finite and again even (residually finite-p) by finite.

d) Let $\pi$ be any set of primes properly containing $\operatorname{spec} M$. Then $G$ has normal subgroups $A \leq H$ with $A$ abelian, $H / A$ residually a finite nilpotent $\pi$ group and $G / H$ finite: $G / A$ is residually finite.

Proof. a) For there is a free abelian subgroup $F$ of $M$ of finite rank with $M / F$ Artinian. The $p$-primary component of $M / F$ is finite, of finite exponent $p^{e}$ say. Let $x \in M$ with $p^{i+e} x \in F$. Then $p^{e} x \in F$ and $p^{i+e} x \in p^{i} F$. Thus $p^{i+e} M \cap F \leq p^{i} F$ and consequently

$$
\left(\bigcap_{i} p^{i} M\right) \cap F \leq \bigcap_{i} p^{i} F=\{0\} .
$$

Therefore $\bigcap_{i} p^{i} M$ embeds into the torsion group $M / F$ and the claim follows.

b) By Part a) here $\bigcap_{i} p^{i} M=\{0\}$. Also each $M / p^{i} M$ is finite. Set $H=$ $=C_{G}(M / p M)$. Then $H$ is normal subgroup of $G$ of finite index; in fact $G / H$ embeds into $G L(n, p)$ for $p^{n}=|M / p M|$. Further $\left[p^{i} M, H\right]=p^{i}[M, H] \leq p^{i+1} M$ for all $i \geq 0$. Thus $H / C_{H}\left(M / p^{i} M\right)$ is a finite $p$-group. Part b) follows.

c) If $H=C_{G}(\{x \in M: p x=0\})$, then $H$ is a normal subgroup of $G$ of finite index and $H$ is a residually a finite $p$-group; for $H$ stabilizes the ascending series

$$
\left\{\left\langle x \in M: p^{i} x=0\right\rangle\right\}_{i \geq 0} .
$$

d) Let $T$ denote the torsion subgroup of $M$ and $T_{p}$ the $p$-primary component of $T$ for the prime $p$. If $T_{p}$ is finite, then $G / C_{G}\left(T_{p}\right)$ is finite. If $T_{p}$ is infinite, then $p \in \operatorname{spec} M$ and $G / C_{G}\left(T_{p}\right)$ is (residually finite- $p$ ) by finite by Part c). By hypothesis there is some prime $p$ in $\pi \backslash \operatorname{spec} M$. By Part b) the group $G / C_{G}(M / T)$ is (residually finite- $p$ ) by finite. Set

$$
A=C_{G}(M / T) \cap \bigcap_{p} C_{G}\left(T_{p}\right)=C_{G}(M / T) \cap C_{G}(T) .
$$

Then $A$ is abelian and $G / A$ is (residually a finite nilpotent $\pi$-group) by finite. Clearly $G / A$ is residually finite.

2.5. Lemma. Let $U$ denote the subgroup of Aut $M$ generated by all its unipotent elements of finite order and let $C$ denote the subgroup of $F_{\infty}$ AutM generated by all its Prüfer subgroups. Set $R=\bigcap_{n} n M$ and let $D$ be the divisible component of the torsion subgroup $T$ of $M$.

a) $[M, U] \leq T$ and $[R, U]=\langle 1\rangle$.

b) $C \leq U$ and $[T, C]=\{0\}$, while $[M, C] \leq D \leq R$.

c) $C$ lies in the centre of $U$ and hence is divisible.

d) $U$ centralizes $[M, C]$ and $C$ centralizes $[M, U]$.

Proof. a) This follows from [11], 2.2a) and 2.4a).

b) If $P$ is Prüfer group, then any irreducible finitary linear image of $P$ is finite dimensional. Now $G L(n, \boldsymbol{Q}), G L\left(n, \boldsymbol{Q}_{p}\right)$ and $G L(n, p)$ contain no Prüfer subgroups, the first two by [8], 9.33, and the third trivially. Then by Theorem $2 b$ ) all Prüfer subgroups of $F_{\infty}$ Aut $M$ are unipotent. Consequently $C \leq U$. That $[T, C]=$ $=\{0\}$ and $[M, C] \leq D$ follow from [5], 13. 
c) Now $C$ embeds by b) into $\operatorname{Hom}(M / T, D)$ via $c \in C$ mapping to $m+T \mapsto$ $\mapsto m(c-1)$, and this embedding is a $U$-embedding. By Part a) this Hom group is centralized by $U$. Therefore $C$ lies in the centre of $U$; clearly then, $C$ is divisible.

d) $U$ centralizes $R \geq D \geq[M, C]$ and $C$ centralizes $T \geq[M, U]$ by Parts a) and $\mathrm{b})$.

2.6. Lemma. Let $G$ be any subgroup of $F_{\infty}$ Aut $M$ and let $R$ and $D$ be as in 2.5 .

a) If $D=\{0\}$, then $M / C_{M}(G)$ is residually finite.

b) $D \leq R$ and $[R, G] \leq D$.

Proof. a) Let $g \in G$. Then $M(g-1)$ is minimax. Since $D=\{0\}$, the torsion subgroup of $M(g-1)$ is finite, so it splits off from $M(g-1)$, e.g. [1], Vol. 1, 27.5. Consequently $M(g-1)$ and hence $M / C_{M}(g)$ are residually finite (by $\left.2.4 \mathrm{a}\right)$ ). It follows that $M / C_{M}(G)$ is residually finite.

b) Clearly $D \leq R$, so $R / D=\bigcap_{n} n(M / D)$. By Part a) we have $R / D \leq$ $\leq C_{M / D}(G)$. That is, $[R, G] \leq D$.

\section{Residual properties.}

3.1. Lemma. Let $G$ be a finitely generated subgroup of $F_{\infty}$ Aut $M$ with $N=$ $=[M, G]$ torsion. Let $\pi=\pi(N)=\{$ primes $p: N$ has an element of order $p\}$. Then $G$ is (residually finite nilpotent $g$-group) by finite.

Proof. Here $N$ is Artinian (so in particular $\pi$ is finite). Then $N=\oplus_{p \in \pi} N_{p}$, where $N_{p}$ is $p$-primary. It suffices to prove the result for $G$ acting on each $M / \oplus_{q \neq p} N_{q}$. Thus we may assume that $N$ is a $p$-group for some prime $p$, necessarily of finite rank, $r$ say. Let $\left\{S_{i}\right\}_{i \geq 0}$ be the upper socle series of $N$, so $S_{i}=$ $=\operatorname{Ann}_{N}\left(p^{i}\right)$ and $\bigcup_{i} S_{i}=N$.

Let $x \in M$. Since $G$ is finitely generated, so $[\boldsymbol{Z} x, G] \leq S_{k}$ for some integer $k$ and then $\boldsymbol{Z} x G \leq \boldsymbol{Z} x+S_{k}$. Hence additively $\boldsymbol{Z} x G$ is a cyclic extension of a finite $p$ group of rank at most $r$. Suppose $x$ has infinite order. Then $\bigcap_{i} p^{i} \boldsymbol{Z} x G=\{0\}$, the group

$$
C_{G}(\boldsymbol{Z} x G / p \boldsymbol{Z} x G) / C_{G}(\boldsymbol{Z} x G)
$$

is residually a finite $p$-group and $\left(G: C_{G}(\boldsymbol{Z} x G / p \boldsymbol{Z} x G)\right) \leq l=|G L(r+1, p)|$. If $x$ is a $p$-element, the same conclusions hold (even with $C_{G}(\boldsymbol{Z} x G / p \boldsymbol{Z} x G) / C_{G}(\boldsymbol{Z} x G)$ a finite $p$-group). Now suppose $x$ is a $p^{\prime}$-element. Then $x(g-1)$ is both a $p$-element and a $p^{\prime}$-element for every $g$ in $G$ and so $G$ centralizes $x$. This trivially the same conclusions also hold in this case.

Let $H$ be the intersection of all the normal subgroups of $G$ of index at most $l$. Since $G$ is finitely generated, so $(G: H)$ is finite. We claim that $H$ is residually a finite $p$-group. Let $h \in H \backslash\langle 1\rangle$. There exists $x$ in $M$ with $x h \neq x$ and we can choose such an $x$ either of infinite order or a $p$-element or a $p^{\prime}$-element. Then $H / C_{H}(Z x G)$ is residually a finite $p$-group. Hence there is a normal subgroup $L$ of $H$ with $H / L$ a finite $p$-group and with $h \notin L$. The result follows. (We can always choose such an $L$ normal in $G$ if we wish, but actually it you chase through the above proof the $L$ you arrive at is automatically normal in $G$.)

3.2. Lemma. Let $G$ be a finitely generated subgroup of $F_{\infty}$ Aut $M$. Then $G$ is abelian by (residually a finite nilpotent $\pi$-group) by finite, for some finite set $\pi$ of primes (depending on $G$ ). 
Proof. Set $C=C_{M}(G)$. By 2.1b) of [2] the group $M / C$ is minimax. Let $X / C$ be the torsion submodule of $M / C$. By 2.4b) the group $G / C_{G}(M / X)$ is (residually finite-nilpotent) by finite.

Now $[X, G]$ is torsion; for if $x \in X$ and $g \in G$ there is a positive integer $m$ with $m x \in C$. Then $m(x(g-1))=(m x)(g-1)=0$, so $x(g-1)$ is torsion and consequently $[X, G]$ is too. It follows from 3.1 that $G / C_{G}(X)$ is (residually finitenilpotent) by finite. Further $A=C_{G}(M / X) \cap C_{G}(X)$ is abelian. The existence of $\pi$ follows from 2.4b) and 3.1; in fact, we can choose $\pi$ to be $\{p\} \cup \pi([X, G])$ for any prime $p$ not in $\operatorname{spec}(M / X)$. Note that

$$
\operatorname{spec}(M / X) \subseteq \operatorname{spec}(M / C)=\operatorname{spec}[M, G] \subseteq \pi([M, G]) .
$$

3.3. Examples. In 6.3 of [6] we construct a minimax group $M$ and a 3-generator soluble subgroup $G$ of Aut $M=F_{\infty}$ Aut $M$ whose centre is a Prüfer 2-group. Clearly $G$ is not residually finite. The wreath product $W$ of $G$ be a cyclic group of order 2 embeds into Aut $(M \oplus M)$ and is not central by residually finite. Of course $W$ is finitely generated and soluble.

3.4. Lemma. Let $G$ be subgroup of $F_{\infty}$ Aut $M$.

a) If $M$ is torsion or residually of finite exponent, the $G$ is residually (finitary over finite images of $\boldsymbol{Z}$ ).

b) If $M$ is torsion-free, then $G$ is abelian by residually (finitary over finite images of $\boldsymbol{Z}$ ).

c) In general $G$ is (nilpotent of class at most 2) by residually (finitary over finite images of $\boldsymbol{Z}$ ). either

Proof. a) Here either $M=\bigcup_{n \geq 1}\{x \in M: n x=0\}$ or $\{0\}=\bigcap_{n \geq 1} n M$. Clearly

$$
\bigcap_{n \geq 1} C_{G}(x \in M: n x=0)=\{0\} \text { or } \bigcap_{n \geq 1} C_{G}(M / n M)=\{0\} .
$$

Part a) follows.

b) Let $R=\bigcap_{n \geq 1} n M$. Then $G / C_{G}(M / R)$ is residually (finitary over finite images of $\boldsymbol{Z}$ ) by a). Also $[R, G]=\{0\} \quad$ by 2.6b). Therefore $C_{G}(M / R)$ here is abelian.

c) Set $S / T=\bigcap_{n \geq 1} n(M / T)$. By 2.6b) again $[S, G] \leq T$. Also $G / C_{G}(M / S)$ and $G / C_{G}(T)$ are residually (finitary over finite images of $\boldsymbol{Z}$ ) by Part a) and hence so is $G / N$ for $N=C_{G}(M / S) \cap C_{G}(T)$. Finally $N$ stabilizes the series $\{0\} \leq T \leq S \leq$ $\leq M$, so $N$ is nilpotent of class at most 2 .

3.5. Examples. Let $A=\left\langle a_{1}, a_{2}\right\rangle$ be a free abelian group of rank 2 , let $B$ be the additive group of the rationals and let $C$ be a Prüfer $p$-group for some prime $p$. Put $M=A \oplus B \oplus C$. Set $X=\operatorname{Hom}(A, B) \cong B^{(2)}, Y=\operatorname{Hom}(B, C)$ and $Z=\operatorname{Hom}(A$, $C) \cong C^{(2)}$. Note that $Y$ is a copy of the additive group of $\boldsymbol{Q}_{p}$ in a natural way. ( $Y$ is torsion-free and divisible, see $G$ ) on p. 182 of Vol. 1 of [1], and is a $Z_{p}$-module via the action on $C$ and hence is naturally a $\boldsymbol{Q}_{p}$-module; finally the exact sequence

$$
0 \rightarrow \boldsymbol{Z} \rightarrow \boldsymbol{Q} \rightarrow \boldsymbol{Q} / \boldsymbol{Z} \rightarrow 0
$$

yields exact sequences

$$
0 \rightarrow \text { End } C \rightarrow Y \rightarrow C \rightarrow 0
$$

and 


$$
0 \rightarrow Z_{p} \rightarrow Y \rightarrow C \rightarrow 0
$$

so $Y \cong \boldsymbol{Q}_{p}$ as $\boldsymbol{Q}_{p}$-module.)

Let $u=\left(\begin{array}{ll}1 & 1 \\ 0 & 1\end{array}\right) \in G L(2, \boldsymbol{Z})$, regarded as an automorphism of $A$ via the given basis $a_{1}, a_{2}$ and let $v$ be a $p$-adic unit (and hence an automorphism of $C$ ) of infinite order. Set

$$
G=\left(\begin{array}{ccc}
\langle u\rangle & X & Z \\
0 & 1 & Y \\
0 & 0 & \langle v\rangle
\end{array}\right),
$$

regarded as a subgroup of Aut $M$ in the obvious way. Let $\xi \in X, \eta \in Y$ and $\zeta \in Z$. Then $M(u-1)=\left\langle a_{2}\right\rangle$ is cyclic, $M \xi=\left\langle a_{1} \xi, a_{2} \xi\right\rangle \leq B$ again is cyclic, $M \eta \leq C$ is Artinian, $M \zeta=\left\langle a_{1} \zeta, a_{2} \zeta\right\rangle$ is finite cyclic and $M(v-1) \leq C$ is Artinian. Therefore $G \leq F_{\infty}$ Aut $M$.

Let $H$ be a normal subgroup of $G$ with $G / H$ periodic. Then $u^{i}$ and $v^{j}$ lie in $H$ for some positive integers $i$ and $j$. Thus $H$ contains $\left\langle u^{i G}\right\rangle$ and hence contains $\left[1+X, u^{i}\right]$. Define $\xi_{1} \in X$ by $a_{1} \xi_{1}=a_{2} \xi_{1}=-i^{-1}$. Then $\xi=\left(u^{-i}-1\right) \xi_{1}$ maps $a_{1}$ to 1 and $a_{2}$ to 0 , which does not depend on $H$, note. Also $1+\xi=1+\left(u^{-i}-\right.$ $-1) \xi_{1}=\left[1+\xi_{1}, u^{i}\right] \in H$. In the same way $H$ contains $\left[1+Y, v^{j}\right]=1+Y\left(v^{j}-1\right)$. Now $v^{j}-1$ is a non-zero element of $\boldsymbol{Z}_{p}$ and hence has an inverse $w$ in $\boldsymbol{Q}_{p}$. Thus $Y w=Y$ and so $Y=Y\left(v^{j}-1\right)$. If $c \in C \backslash\{0\}$ there is an element $\eta$ of $Y$ with $1 \eta=$ $=c$. (Note the wide choice for $c$ available here; we will make more specific choices for $c$ later.) Then the periodic residual $N=\bigcap_{H} H$ of $G$ contains $1+\xi$ and $1+\eta$. A simple calculation shows that $[1+\xi, 1+\eta]=1+\xi \eta \in 1+Z$ and $a_{1} \xi \eta=1 \eta=$ $=c \neq 0$. Therefore $N$ is not abelian.

Finitary groups over finite images of $\boldsymbol{Z}$ are locally finite (e.g. [5], 11). Thus by $3.4 \mathrm{c}$ ) we have $[N, N, N]=\langle 1\rangle$. We now show that in general (that is, for some subgroups $G$ of $F_{\infty}$ Aut $M$ with periodic residuals $N$ ) neither $[N, N, G]$ nor $[N, G$, $N]$ nor $[[N, G],[N, G]]$ need be $\langle 1\rangle$; in particular the periodic residual of $G$ need not be central (in $G$ ) by abelian nor abelian by central (in $G$ ).

We may choose $c$ in the above construction with $c v \neq c$. Then $\xi \eta v \neq \xi \eta$. Since $[1+\xi, 1+\eta]^{\operatorname{diag}(1,1, v)}=1+\xi \eta v$, the element $[1+\xi, 1+\eta]$ does not lie in the centre of $G$. Then with this choice of $c$ we have $[N, N, G] \neq\langle 1\rangle$. Now

Now choose any $c$ with $|c|>4$, and we can do this with $c v \neq c$ if we wish.

$$
\begin{gathered}
{[1+\xi, \operatorname{diag}(-1,1,-1)]=1-2 \xi,} \\
{[1+\eta, \operatorname{diag}(-1,1,-1)]=1-2 \eta,} \\
{[1-2 \xi, 1-2 \eta]=1+4 \xi \eta}
\end{gathered}
$$

and

$$
a_{1}(4 \xi \eta)=4 c \neq 0 .
$$

Set $G_{0}=\langle G$, diag $(-1,1,-1)\rangle$. Since $[M$, diag $(-1,1,-1)]=2 A \oplus C$, so $G_{0} \leq$ $\leq F_{\infty}$ Aut $M$. The periodic residual $N_{0}$ of $G_{0}$ contains $N$ and

$$
\left[N_{0}, G_{0}, N_{0}\right] \geq\left[\left[N_{0}, G_{0}\right],\left[N_{0}, G_{0}\right]\right] \geq\left[\left[N, G_{0}\right],\left[N, G_{0}\right]\right] \neq\langle 1\rangle .
$$


Thus our second and third claims are also substantiated.

3.6. Lemma. Let $G \leq F_{\infty}$ Aut $M$ and set $A=C_{G}(M / T) \cap C_{G}(T)$. Then $A$ is abelian and unipotent and $G / A$ is residually (finitary over $Q$ and finite images of $\boldsymbol{Z}$ ).

Proof. The group $G / C_{G}(M / T)$ embeds into $F G L(Q \otimes M)$ and so is actually finitary over $\boldsymbol{Q}$. Also $G / C_{G}(T)$ is residually (finitary over finite images of $\boldsymbol{Z}$ ) by 3.4a). The claim follows.

3.7. Examples. In 3.6 we cannot always find a central subgroup of $G$ modulo which $G$ is residually finitary.

For let $M$ and $G \leq$ Aut $M$ be as in $\mathbf{6 . 3}$ of [6]. Then $M$ is minimax, so $G \leq$ $\leq F_{\infty}$ Aut $M$. Also $G$ is finitely generated and has a central Prüfer subgroup, $Z$ say. If $\rho: G \rightarrow F \operatorname{Aut}_{R} N$ is a homomorphism for some module $N$ over any commutative ring $R$, then $G \rho$ is residually finite $([6], \mathbf{6 . 2 b}))$, so $Z \rho=\{0\}$. Thus $G$ is not residually (finitary over commutative rings). The same argument (and [6], 6.2c)) shows that $G$ is not residually (Artinian-finitary over commutative rings) either. The offending subgroup in both cases, namely $Z$, is central in $G$. The wreath product $W$ of $G$ by a cyclic group of order 2 embeds into $F_{\infty}$ Aut $M^{(2)}$ and the intersection of all normal subgroups $N$ of $W$ with $W / N$ either finitary of Artinian-finitary over some commutative ring, is abelian and normal but not central in $W$.

3.8. Proof of Theorem 1. Part a) of Theorem 1 follows from 2.1b) \& 3.2, Part b) from 3.4 and [5], 11 (if $M$ has finite exponent, then $F_{\infty}$ Aut $M$ is always locally finite) and Part c) from 3.6. The examples mentioned in the Introduction, preventing various generalizations or extensions of Theorem 1, are given in 3.3, 3.5 and 3.7.

3.9. Proof of Theorem 3. Set $B=C_{G}(M / T) \cap C_{G}(T)$. Clearly $B$ is abelian and normal in $G$. Let $T=\oplus_{p} T_{p}$, where $T_{p}$ is the $p$-primary component of $T$. Then $G / B$ embeds into

$$
\left(G / C_{G}(M / T)\right) \times\left(\times_{p}\left(C_{G}\left(T_{p}\right)\right)\right),
$$

and $I$ do mean direct product here and not just cartesian product. $G / C_{G}(M / T)$ embeds naturally into $F G L(Q \otimes M)$ and $G / C_{G}\left(T_{p}\right)$ embeds naturally into $F_{1}$ Aut $T_{p}$. If $g \in G$, then $g$ is unipotent if and only if the images of $g$ in $F G L(Q \otimes$ $\otimes M)$ and the $F_{1}$ Aut $T_{p}$ are unipotent. Thus it suffices to prove the result just in the two cases where either $M$ is torsion-free or $M$ is a $p$-group for some prime $p$.

Suppose $M$ is torsion-free, so $G \leq F G L(Q \otimes M)$. Set $K=\langle 1\rangle=B$. Here $u(G)$ is torsion-free. Thus the first part of c) is trivial in this case; the second part of c) is given by 3.4b). Part d) here is trivial with $\boldsymbol{Q}$ the only field arising, as is Part e). Part f), of course, is totally trivial.

Now assume that $M$ is a $p$-group. Then $M$ is a module in the obvious way over the local ring of $\boldsymbol{Z}$ at $p$. Hence Section 7 of [9] is applicable. Let

$$
V=M[p]=\{x \in M: p x=0\}
$$

and set $H=C_{G}(V)$ and $K=H \cap u(G)$. By [9], 7.1, the group $H$ is residually (a nilpotent $p$-group of finite exponent) and $G / H$ embeds naturally into the finitary linear group $F G L(V)$ over $G L(p)$. Also $u(G)$ is residually a $p$-group and $H$ stabilizes an ascending series in $M$, cf. the proof of [9], 7.3. We now have a), b), c) d) and $\mathrm{f}$ ). By $\mathrm{b}$ ) of Theorem 2 (which we have already proved, see 2.2) the group $G / u(G)$ is a subdirect product of finitary linear groups over $Q, G F(p)$ and $\boldsymbol{Q}_{p}$ (only for the one prime $p$ ). Since $G / K$ embeds into $G / H \times G / u(G)$, so Part e) follows. 
A similar argument yields the following.

3.10. Theorem. Let $G$ be a subgroup of $F_{\infty}$ Aut $M$. Then $G$ has a normal series

$$
\langle 1\rangle \leq B \leq K \leq s(G) \leq G
$$

where

a) $B$ is abelian,

b) $K / B$ is residually (nilpotent of finite exponent),

c) $s(G)$ is the $s$-radical of $G$ and is locally residually nilpotent,

d) $s(G) / B$ is a subdirect product of a torsion-free Fitting group and a residually (periodic Fitting group); it is also abelian by residually (a periodic Fitting group),

e) $s(G) / K$ is a subdirect product of unipotent finitary linear groups over $Q$ and $G F(p)$ for various primes $p$,

f) $G / K$ is a subdirect product of finitary linear groups over $Q$ and $G F(p)$ for various primes $p$,

g) $G / s(G)$ is a subdirect product of irreducible finitary linear groups over $Q$ and $G F(p)$ for various primes $p$,

h) $K$ stabilizes an ascending series in $M$.

Proof. Again set $B=C_{G}(M / T) \cap C_{G}(T)$. A torsion-free $Z$-module is tight, so any $s$-subgroup of $G$ acts unipotently on $M / T$ by $\mathbf{4 . 3}$ of [2]. Thus again we may assume that $M$ is either torsion-free or a $p$-group for some prime $p$. If $M$ is torsionfree set $K=\langle 1\rangle$. Here $s(G)=u(G)$ and the claims follow easily in this case.

Now assume that $M$ is a $p$-group. Set $B=\langle 1\rangle, V=M[p]$ and $K=C_{G}(V)$. Then $K$ satisfies h), cf. the proof of [9], 7.3, and b) by [9], 7.1. In particular $K \leq s(G)$. The inverse image in $G$ of the unipotent radical of $G$ on $V$ acts unipotently on $V_{i}=$ $=\left\{x \in M: p^{i} x=0\right\}$ for each $i$ and clearly $s(G)$ acts unipotently on $V$. Thus this inverse image is $s(G)$. Also $G / K$ embeds into $F G L(V)$ and the above shows that $s(G) / K$ maps onto the unipotent radical of this image of $G / K$. In particular this yields e). Part c) is part definition and Part a) consequence of [2], 4.6. Also $s(G)$ is residually a Fitting $p$-group; this can be seen directly, alternatively cf. [9], 7.3. The second part of d) is then trivial. $G / s(G)$ is a subdirect product of irreducible finitary linear groups by 4.1 of [6]. A check of proof of that shows that the only ground field that arises in this case is $G F(p)$. This proves $\mathrm{g}$ ). Finally $\mathrm{f}$ ) follows from the action of $G$ on $V$.

\section{Unipotent subgroups.}

4.1. Lemma. Let $E$ be the intersection of all subgroups $X$ of $M$ with $M / X$ divisible and let $G$ be a unipotent subgroup of Aut $M$.

a) $E$ has no non-zero divisible image.

b) $D \cap E=\{0\}$ for $D$ the divisible component of $T$.

c) $G / C_{G}(M / E)$ and $G / C_{G}(D)$ are torsion-free.

d) $F_{\infty}$ Aut $E=F$ Aut $E$.

e) If $G \leq F_{\infty}$ Aut $M$, then $G / C_{G}(T / D)$ and $G / C_{G}(E \cap T)$ are periodic.

Proof. a) If $E$ is divisible, then $M=E \oplus X$ for some $X$ and then $M / X$ is divisible. Hence $E \leq X$ and $E=\{0\}$. Part a) follows from this.

b) Also $M=D \oplus X$ for some $X$, so $M / X$ is divisible, $E \leq X$ and $D \cap E=\{0\}$.

c) Let $g \in G$ have finite order on $M / E$. Then $M(g-1)$ modulo $E$ has finite exponent by [9], 2.1. Hence if $M / X$ is divisible, then $E \leq X$, so $(M / X)(g-1)$ is 
divisible of finite exponent and hence is $\{0\}$. Thus $M(g-1) \leq \bigcap_{X} X=E$ and $g \equiv$ $\equiv 1$ modulo $E$.

Now assume that $g \in G$ has finite order on $D$. Then $D(g-1)$ is divisible of finite exponent and hence $D(g-1)=\{0\}$. We have now shown that $G / C_{G}(M / E)$ and $G / C_{G}(D)$ are torsion-free.

d) Let $g \in F_{\infty}$ Aut $E$. Then $E / C_{E}(g) \cong E(g-1)$ is minimax with (by a) no nonzero divisible image. Hence $E(g-1)$ is finitely generated and so $g \in F$ Aut $E$. It follows that $F_{\infty}$ Aut $E=F$ Aut $E$.

e) Here $G \leq F_{\infty}$ Aut $M$. Let $g \in G$. Then $(T / D)(g-1)$ is a periodic minimax group with zero divisible component. Thus $(T / D)(g-1)$ is finite and consequently $g$ has finite order on $T / D$, e.g. by [9], 2.2c). Therefore $G / C_{G}(T / D)$ is periodic. Finally $G / C_{G}(E \cap T)$ is periodic by d) and [9], 2.2c) again.

4.2. Proof of Theorem 4a). Let $P$ be the maximal periodic subgroup of $G$. Using the notation of 4.1 we have from 4.1c) and [9], 2.2a) that

$$
P \leq C_{G}(M / E) \cap C_{G}(M / T)=C_{G}(M /(E \cap T)) .
$$

Set $Q=C_{P}(T / D)$. Now $[E \cap T, Q] \leq D \cap E=\{0\}$ by 4.1b). Thus $Q$ stabilizes the series $M \geq E \cap T \geq\{0\}$ and consequently $Q$ is periodic and abelian. Also $G / Q$ embeds into

$$
(G / P) \times\left(G / C_{G}(T / D)\right),
$$

so $G / Q$ is torsion-free by periodic, see $4.1 \mathrm{e}$ ).

4.3. Example. Let $M=\boldsymbol{Z} \oplus P$, where $P$ is the direct sum of two Prüfer $p$ groups, for some prime $p$. Then $M$ is minimax and it is easy to see that $F_{\infty}$ Aut $M=$ $=$ Aut $M$ contains a unipotent subgroup $G$ isomorphic to the split extension of $P$ by the subgroup of Aut $P$ generated by $\left(\begin{array}{ll}1 & 0 \\ 1 & 1\end{array}\right)$. Further it is easy to see that $G$ is not torsion-free by periodic, so the abelian normal subgroup of Theorem 4a) cannot be eliminated.

4.4. Proof of Theorem 4b). By Theorem 2c) we have $U \in N$ and by 2.5a) (and 2.1a)) we have $[M, U] \leq T$ with $U / C_{U}(T)$ embeddable into $F_{1}$ Aut $T$. We apply the proof of [9], 4.4, to $T$. In doing so we construct a series

$$
M \geq T=N_{0} \geq N_{1} \geq \ldots \geq N_{r} \geq N \geq\{0\}
$$

of finite length stabilized by $U$ with $T / N_{r}$ of finite exponent and $N=\bigcap_{n} n T$; note that $[N, U]=\{0\}$ by 2.5a) again.

Set $V=C_{U}(T / N)$. Then $V$ stabilizes the series $M \geq T \geq N \geq\{0\}$, so $V \in N_{2}$. Also $U / V$ stabilizes the series $N_{0} \geq N_{1} \geq \ldots \geq N_{r} \geq N$ with $N_{0} / N_{r}$ of finite exponent. Thus $U / V \in \boldsymbol{E}$ and hence $U \in N_{2} \boldsymbol{E}$.

Now set $W=C_{U}\left(N_{r}\right) \cap C_{U}\left(M / N_{r}\right)$. Here $W$ stabilizes $M \geq N_{r} \geq\{0\}$, so $W$ is abelian. Similarly $U / C_{U}\left(N_{r}\right)$ is abelian since it stabilizes $N_{r} \geq N \geq\{0\}$. Finally $U / C_{U}\left(M / N_{r}\right)$ stabilizes $M \geq N_{0} \geq \ldots \geq N_{r}$ with $N_{0} / N_{r}$ of finite exponent, so $U / C_{U}\left(M / N_{r}\right) \in \boldsymbol{E}$. Consequently $U / W \in S(\boldsymbol{A} \times \boldsymbol{E})$ and $U \in \boldsymbol{A} \cdot S(\boldsymbol{A} \times \boldsymbol{E})$. Trivially then, $U \in \boldsymbol{A} \boldsymbol{E} \boldsymbol{A}$.

4.4. Proof of Theorem 4c). This is actually a simple corollary of Theorem 4b). 
If $X$ is a finite subset of $G$, then $\left\langle X^{G}\right\rangle \in N \cap N_{2} \boldsymbol{E} \cap \boldsymbol{A} \boldsymbol{E} \boldsymbol{A}$ by Theorem 4b). If $Y$ is a finite subset of $G^{\prime}$, then $Y$ lies in $\langle X\rangle^{\prime}$ for some finite subset $X$ of $G$. Hence $\left\langle Y^{G}\right\rangle \leq\left\langle X^{G}\right\rangle^{\prime}$ and so $\left\langle Y^{G}\right\rangle \in \boldsymbol{N} \cap \boldsymbol{A} \boldsymbol{E}$.

5. Maximin groups; a variant. Instead of minimax groups, suppose we consider abelian groups that are extensions of Artinian groups by Noetherian groups. Such a group is just

(*) a direct sum of a finite number of cyclic and Prüfer groups.

This class does not fit into the general framework of [2], but does still define a finitary group (cf. [3], Section 2); namely

$$
F_{A+N} \text { Aut } M=\{g \in \text { Aut } M: M(g-1) \text { satisfies (*) above }\} .
$$

The suffix $A+N$ here is suggested by the fact that an abelian group satisfies (*) if and only if it is a sum of an Artinian group and a Noetherian group. We coin the term maximin group for such a group, a name that seems not to have been used before. (In contrast the term minimax group has been in used for some forty years at least.)

Clearly $\left\langle F\right.$ Aut $M, F_{1}$ Aut $\left.M\right\rangle \leq F_{A+N}$ Aut $M \leq F_{\infty}$ Aut $M$ for any abelian group $M$. Thus Theorems 1 to 4 trivially apply to $F_{A+N}$ Aut $M$ and its subgroups. Further the counter examples constructed in 2.3, 3.5 and 4.3 all lie in $F_{A+N}$ Aut $M$. This in not the case for the examples constructed in 3.3 and 3.7, which are based on the construction of [6], 6.3. This raises the possibility that Parts a) and c) of Theorem 1 can be strengthened for $F_{A+N}$ Aut $M$. In both cases they can.

Theorem 5. Let $M$ be an abelian group with torsion subgroup $T$. Set $G=$ $=F_{A+N}$ Aut $M$.

a) $A=C_{G}(M / T) \cap C_{G}(T)$ is an abelian normal subgroup of $G$ and $G / A$ embeds into the direct product $F \operatorname{Aut}(M / T) \times F_{1}$ Aut $T$. There is a natural exact sequence

$$
1 \rightarrow F_{1} \text { Aut } M \rightarrow F_{A+N} \text { Aut } M \rightarrow F \text { Aut }(M / T) .
$$

b) $G$ is locally residually finite.

c) $G$ is unipotent-abelian by residually (finitary over $\boldsymbol{Z}$ ).

5.1. Lemma. Let $G$ be a finitely generated subgroup of $\mathrm{Aut}_{R} M$, where $M$ is a module over the commutative ring $R$. Suppose $[M, G] \leq N+A$, where $N$ is a Noetherian $R$-submodule of $M$ and $A$ is an Artinian $R$-submodule of $M$. If $X$ is a finite subset of $M$, then $R X G=\sum_{x \in X, g \in G} R x g$ is finitely generated as $R$-module.

Proof. If $A_{+}$denotes the sum of all the Artinian $R$-submodules of $N+A$, then $A \leq A_{+}$and $A_{+} / A$ is $R$-Noetherian. It follows that $A_{+} / A$ and hence $A_{+}$are $R$ Artinian. Hence we may assume that $A=A_{+}$. Clearly then $A G \leq A$. Define $A_{i}$ inductively by letting $A_{0}=\{0\}$ and letting $A_{i+1} / A_{i}$ denote the socle of the $R$ module $A / A_{i}$. Then $\bigcup_{i \geq 0} A_{i}=A$, each $A_{i}$ is Noetherian (as well as Artinian) and $A_{i} G \leq A_{i}$ for each $i$.

Suppose $X=\left\{x_{1}, x_{2}, \ldots, x_{l}\right\}, N=R y_{1}+R y_{2}+\ldots+R y_{m}$ and $G=\left\{g_{1}, g_{2}, \ldots\right.$ $\left.\ldots, g_{n}\right\}$. There exists an integer $t$ such that for all $i, j$ and $k$ we have $x_{i}\left(g_{k}-1\right) \in$ $\in N+A_{t}$ and $y_{j}\left(g_{k}-1\right) \in N+A_{t}$. In particular $N g_{k} \leq N+A_{t}$ for each $k$ and so $(N+$ $\left.+A_{t}\right) G \leq N+A_{t}$. But then the $g_{k}$ and hence each element of $G$ centralize $R X$ modulo $N+A_{t}$. Consequently $R X G \leq R X+N+A_{t}$ and so $R X G=R X+\left(\left(N+A_{t}\right) \cap\right.$ $\cap R X G)$. But $N+A_{t}$ is Noetherian, so $\left(N+A_{t}\right) \cap R X G$ is too, and $X$ is finite by hypothesis. Therefore $R X G$ is finitely generated. 


\subsection{Proof of Theorem 5.}

a) As in the proof of 2.1 we have $F_{1}$ Aut $M=C_{G}(M / T)$. Clearly $A$ is abelian, $F_{A+N} \operatorname{Aut}(M / T)=F \operatorname{Aut}(M / T)$ and $F_{A+N} \operatorname{Aut} T=F_{1}$ Aut $T$. Both claims in a) follow from this.

b) Let $H$ be a finitely generated subgroup of $G$ and apply 5.1 to $H$ with $R=Z$. If $X$ is any finite subset of $M$, then $Z X H$ is finitely $Z$-generated. Hence $H / C_{H}(Z X H)$ is residually finite. (In fact $H / C_{H}(Z X H)$ for every prime $p$ is (residually a finite $p$-group) by finite.) Part b) follows.

c) $A$ is unipotent and abelian, trivially $F \operatorname{Aut}(M / T)$ is finitary over $Z$ and $F_{1}$ Aut $T$ is residually (finitary over finite images of $\boldsymbol{Z}$ ), e.g. by [9], 8.3. Part c) follows.

If $M$ itself is maximin, we can squeeze a little more out of this.

5.3. Remark. Let $H$ be a finitely generated subgroup of $G=$ Aut $M$, where $M$ is an abelian maximin group.

a) $G$ need not be residually finite and need not be quasilinear.

b) $H$ is isomorphic to a linear group of finite degree over the complex numbers $\boldsymbol{C}$.

c) For all but a finite set of primes $p$ the group $H$ is (residually a finite $p$-group) by finite.

Proof. a) If $K$ is the split extension of a Prüfer $p$-group $P$ by the group $U$ of units of the ring of $p$-adic integers, then $K$ embeds into Aut $(\boldsymbol{Z} \oplus P)$ and is not residually finite and is not quasilinear, see [6], 5.2.

b) $M=F \oplus D$, where $F$ is finitely generated and $D$ is divisible and Artinian. Thus we may take Aut $M$ in the form

$$
\left(\begin{array}{cc}
\text { Aut } F & \operatorname{Hom}(F, D) \\
0 & \text { Aut } D
\end{array}\right) .
$$

Hence $H \leq\left\langle X, Y, Z_{0}\right\rangle$, where $X$ is a finitely generated subgroup of Aut $F \oplus 1_{D}, Y$ is a finitely generated subgroup of $1_{F} \oplus$ Aut $D$ and $Z_{0}-1$ is a finite subset of $\operatorname{Hom}(F, D)$. There exists an integer $n$ such that if $D_{n}=\{x \in D: n x=0\}$, then $Z_{0}-$ -1 lies in $\operatorname{Hom}\left(F, D_{n}\right)$. Set $Z=1+\operatorname{Hom}\left(F, D_{n}\right)$. Then $Z$ is a finite normal subgroup of $H_{0}=X Y Z$ and $H \leq H_{0}$. Clearly $\left(H_{0}: X Y\right)$ is finite and $X Y$ is isomorphic to a linear group of finite degree over $C$, consequently so too are $H_{0}$ and $H$.

c) This follows from Part b) and 4.7 of [11].

Suppose $M$ is torsion-free. Then $F_{\infty}$ Aut $M$ is finitary over $\boldsymbol{Q}$ (see 2.1) and abelian by (residually finitary over finite images of $\boldsymbol{Z}$ ) by 3.4b). Further $F_{A+N}$ Aut $M$ is actually finitary over $\boldsymbol{Z}$. However $F_{\infty}$ Aut $M$ need not be finitary over $\boldsymbol{Z}$ or even residually (finitary over $\boldsymbol{Z}$ ) as we show in 5.6 below. In general, that is with $M$ not necessarily torsion-free, while $F_{\infty}$ Aut $M$ is (nilpotent of class $\leq 2$ ) by residually (finitary over finite images of $\boldsymbol{Z}$ ) by 3.4c) and while $F_{A+N}$ Aut $M$ is abelian by residually (finitary over $\boldsymbol{Z}$ ) by Theorem $5, F_{\infty}$ Aut $M$ need not be abelian by residually (finitary over $\boldsymbol{Z}$ ). This too we show below, see 5.7. We need a little preparation.

5.4. Example. Let $G=\left\langle a, b \mid a^{b}=a^{q}\right\rangle$, where $q$ is some prime. Then $G$ is isomorphic to the subgroup

$$
\left\langle\left(\begin{array}{ll}
1 & 0 \\
1 & 1
\end{array}\right),\left(\begin{array}{ll}
q & 0 \\
0 & 1
\end{array}\right)\right\rangle
$$

of $G L(2, Z[1 / q])$. Thus if $M_{1}$ is the direct product of two Prüfer $p$-groups for any 
prime $p \neq q$, then $G$ embeds into $F_{A+N}$ Aut $M_{1}=F_{1}$ Aut $M_{1}=$ Aut $M_{1}$. If $M_{2}$ is a free $Z[1 / q]$-module of rank 2 , then $M_{2}$ is $Z$-torsion-free and $G$ embeds into $F_{\infty}$ Aut $M_{2}=$ Aut $M_{2}$.

However $G$ does not embed into $F$ Aut $M$ for any abelian group $M$, for if it did we would have $G$ acting faithfully on some finitely generated subgroup of $M$ by [4], 2.3c), so $G$ would embed into $G L(n, \boldsymbol{Z})$ for some integer $n$. But $G L(n, \boldsymbol{Z})$ is (residually a finite $q$-group) by finite, while $G$ is not; indeed its subgroup $\left\langle a^{G}\right\rangle$ is isomorphic to the additive group of $Z[1 / q]$. However $G$ is residually finite and hence is trivially residually (finitary over finite images of $\boldsymbol{Z}$ ).

5.5. Example. Let $p \neq q$ be primes and let $G=\langle g\rangle P$ be the extension of the Prüfer $p$-group $P$ by its automorphism $g: h \mapsto h^{q}$. Then $G$ does not embed into $F$ Aut $M$ for any abelian group $M$.

Proof. Suppose $G \leq F$ Aut $M$. Then $[M, P] \leq D \leq T$ and $[T, P]=\{0\}$ by 2.5b). Hence $N=T \cap M(g-1)$ is finite and centralized by $P$. Thus $N$ is a $G$ submodule of $M$ and $C_{P}(M / N)$ is finite. Therefore $P / C_{P}(M / N)$ is isomorphic to $P$ and so $G / C_{G}(M / N)$ is isomorphic to $G$. Consequently we may pass to $M / N$ and assume that $N=\{0\}$. In particular now $T(g-1)=\{0\}$ and so $[T, G]=\{0\}$.

Let $x \in M$ and $h \in P$. Then $h^{g}=h^{q}$ and so

$$
x g^{-1}(h-1)=x\left(h^{g}-1\right)=x\left(h^{q}-1\right)=q x(h-1),
$$

where we have used $[M, P] \leq T$ and $[T, G]=\{0\}$. Thus $x g^{-1}$ is congruent to $q x$ modulo $C_{M}(h)$ for any $h$ in $P$, so in fact $x g^{-1}$ is congruent to $q x$ modulo $C_{M}(P)$.

Set $M^{*}=M / C_{M}(P)$. Then $(q-1) M^{*}=M^{*}\left(g^{-1}-1\right)$, which is finitely generated. Also $M^{*}$ and hence $(q-1) M^{*}$ is $q$-divisible. Thus $(q-1) M^{*}$ is finite (even a finite $q^{\prime}$-group). Hence $M^{*}$ has finite exponent (prime to $q$ ). But $P$ stabilizes the series $M \geq C_{M}(P) \geq\{0\}$, so $P$ embeds into the group $\operatorname{Hom}\left(M^{*}\right.$, $\left.C_{M}(P)\right)$, which also has finite exponent. This is manifestly false and 5.5 follows.

5.6. Example. Let $G=\langle A, b| a^{b}=a^{q}$ for all $a$ in $\left.A\right\rangle$, where $A$ is a copy of the additive group of $\boldsymbol{Q}$ and $q$ is a prime.

a) $G$ embeds into $F_{\infty} \operatorname{Aut}(\boldsymbol{Z}[1 / q] \oplus \boldsymbol{Q})$.

b) $G$ is not residually periodic; indeed $A \neq\langle 1\rangle$ is the periodic residual of $G$.

c) $G$ is not residually (finitary over $\boldsymbol{Z}$ ) and $A$ is the (finitary over $\boldsymbol{Z}$ ) residual of $G$.

Note that in a) the abelian group $Z[1 / q] \oplus Q$ is torsion-free. Trivially both b) and c) imply that $G$ is not residually (finitary over finite images of $\boldsymbol{Z}$ ).

Proof. a) Clearly $G$ is isomorphic to the subgroup

$$
\left\langle\left(\begin{array}{cc}
q & 0 \\
0 & 1
\end{array}\right),\left(\begin{array}{cc}
1 & \phi_{r} \\
0 & 1
\end{array}\right): r \in \boldsymbol{Q}\right\rangle
$$

of $F_{\infty} \operatorname{Aut}(Z[1 / q] \oplus \boldsymbol{Q})$, where $\phi_{r}$ denotes the homomorphism of $Z[1 / q]$ into $\boldsymbol{Q}$ given by $1 \mapsto r$.

b) Suppose $N$ is a normal subgroup of $G$ with $G / N$ periodic and $A$ not contained in $N$. Then $A N / N$ is nontrivial, periodic and divisible. Also $b$ acts on $A N / N$ by raising elements to their $q$-th powers. Hence $G / N$ contains a copy of the group $G$ of 5.5 for some prime $p \neq q$. This group is not periodic. It follows easily that $A$ lies in, and hence is, the periodic residue of $G$. 
c) Now suppose that $N$ is the kernel of a homomorphism of $G$ into some $F$ Aut $M$. Assume $\langle 1\rangle \neq A \cap N \neq A$. Then again $A N / N$ is periodic and $G / N$ contains a copy of the group $G$ of 5.5 for some prime $p \neq q$. This contradicts 5.5 and therefore either $A \cap N=\langle 1\rangle$ or $A \leq N$.

Suppose $A \cap N=\langle 1\rangle$. Since $C_{G}(A)=A$, this implies that $N=\langle 1\rangle$. But $G$ contains a copy of the group $G$ of 5.4. This contradicts the conclusion of 5.4 that its group embeds into no finitary group over $\boldsymbol{Z}$. Therefore $A \leq N$. It follows easily that $A$ is the (finitary over $\boldsymbol{Z}$ ) residual of $G$.

5.7. Example. Let $M=Z[1 / q] \oplus B \oplus C$, where $B$ is the additive group of $\boldsymbol{Q}$, where $C$ is a Prüfer $p$-group and where $p$ and $q$ are distinct primes. Set

$$
G=\left(\begin{array}{ccc}
\langle q\rangle & X & Z \\
0 & 1 & Y \\
0 & 0 & \langle q\rangle
\end{array}\right) \leq F_{\infty} \text { Aut } M,
$$

where $X=\operatorname{Hom}(Z[1 / q], B) \cong B, Y=\operatorname{Hom}(B, C) \cong Q_{p}$ and $Z=\operatorname{Hom}(Z[1 / q], C)$ $\cong C$. Note that $q$ is a unit of $\boldsymbol{Z}_{p}=$ End $C$. Let $N$ denote the (finitary over $\boldsymbol{Z}$ ) residual of $G$. Then $N$ contains $\langle 1+X, 1+Y\rangle$. In particular $G$ is not abelian by residually (finitary over $\boldsymbol{Z}$ ).

Proof. Firstly it is easy to check that $G$ lies in $F_{\infty}$ Aut $M$. Now apply 5.6c) to the groups

$$
\left(\begin{array}{cc}
\langle q\rangle & X \\
0 & 1
\end{array}\right) \text { and }\left(\begin{array}{cc}
1 & Y \\
0 & \langle q\rangle
\end{array}\right) .
$$

We deduce that $N$ contains $1+X$ and $1+Y$. If $\xi$ denotes the natural embedding of $Z[1 / q]$ into $B=Q$ and if $\eta$ is any homomorphism of $B$ into $C$ with $1 \eta \neq 0$, then $1 \xi \eta \neq 0$ and $\xi \eta \neq 0$. But $[1+\xi, 1+\eta]=1+\xi \eta \neq 1$. Therefore $N$ is not abelian. (Necessarily by 3.4c) $N$ is nilpotent of class 2.)

1. Fuchs L. Infinite abelian groups (2 vols). - New York: Acad. Press, 1970, 1973.

2. Wehrfritz, B. A. F. On generalized finitary groups // J. Algebra. - 2002. - 247. - P. $707-727$.

3. Wehrfritz B. A. F. Finitary and Artinian-finitary groups. - Preprint.

4. Wehrfritz B. A. F. Finitary automorphism groups over commutative rings // J. Pure and Appl. Algebra. - 2002. - 172. - P. 337-346.

5. Wehrfritz B. A. F. Finitary and Artinian-finitary groups over the integers $\mathbf{Z} / /$ Ukr. Math. J. 2002. - 54, № 7. - P. 924-936.

6. Wehrfritz B. A. F. Artinian-finitary groups over commutative rings // Ill. J. Math. - 2003. - 47. P. $551-565$.

7. Wehrfritz B. A. F. Finitary and Artinian-finitary groups over commutative rings // J. Group Theory. - 2004. - 7. - P. 243-253.

8. Wehrfritz, B. A. F. Artinian-finitary groups over commutative rings and non-commutative rings // J. London Math. Soc. - 2004. - 70. - P. 325-340.

9. Wehrfritz B. A.F. The similarity between finitary and Artinian-finitary groups // Monatshefte für Mathematik (to appear).

10. Wehrfritz B. A. F. Finitary automorphism groups over non-commutative rings // J. London Math. Soc. - 2001. - 64. - P. 611-623.

11. Wehrfritz B. A. F. Infinite linear groups. - Berlin: Springer, 1973. 